\section{PSQ-109 READMISSIONS OF OLDER PATIENTS PRESENTING TO HOSPITAL WITH A FALL (RELIEF): A SYSTEMATIC REVIEW}

${ }^{1} \mathrm{C}$ Ratsimbazafy ${ }^{*}{ }^{2} \mathrm{C}$ Schwab, ${ }^{3} \mathrm{~A}$ Dechartres, ${ }^{2} \mathrm{C}$ Fernandez, ${ }^{2} \mathrm{P}$ Hindlet. ${ }^{1} \mathrm{Aphp}$ Sorbonne Université, Pharmacy, Paris, France; ${ }^{2}$ Aphp Sorbonne Université-Inserm-Institut Pierre Louis D'épidémiologie et de Santé Publique, Pharmacy, Paris, France; ${ }^{3}$ Aphp Sorbonne UniversitéService De Biostatistique Santé Publique Information Médicale-Sorbonne Université-InsermInstitut Pierre Louis D'epidémiologie et de Santé Publique, Biostatistics Public Health Medical Information, Paris, France

\subsection{6/ejhpharm-2020-eahpconf.426}

Background and importance Falls are an important issue in the elderly as they are frequent, deleterious and often lead to hospitalisation. Hospitalisation increases the occurrence of adverse events, including unplanned readmissions.

Aim and objectives Our principal objective was to identify interventions designed to prevent unplanned readmissions or emergency department (ED) visits of elderly patients presenting to hospital with a fall. Our secondary objectives were to assess whether these interventions decreased fall recurrence and to detect any harm or unintended effects of these interventions.

Material and methods On 11 February 2019, we performed a systematic review in MEDLINE via PubMed, EMBASE, Cochrane Central Register of Controlled Trials and Web of Science, without date or language restrictions. We manually updated this search on 1 August 2019. Study selection and data extraction were performed independently by two reviewers. We included all studies reporting interventions to prevent unplanned readmissions or ED visits of older patients (aged $\geq 65$ years) presenting to hospital with a primary diagnosis of a fall (PROSPERO registration No: CRD42019131965)

Results We identified 475 unique citations after removing duplicates and 7 studies were included ( 2 observational and 5 interventional studies, published between 2010 and 2019), reporting heterogeneous interventions. The evaluated intervention was shown to be effective in three studies, reducing readmissions or ED revisits (35-58\%) compared with the control groups. In these studies, interventions were multifaceted: (1) multidisciplinary assessment in a geriatric ward and referral to health community services, (2) brief patient education in the ED by an ergotherapist and a physiotherapist and (3) clinical pharmacy activities by a pharmacy resident in a geriatric emergency unit. Regarding our secondary objectives, only three studies assessed the reduction in fall recurrence and the results were not significant; no study assessed harm or unintended effects caused by the interventions.

Conclusion and relevance Despite relatively heterogeneous interventions, our systematic review identified diverse intervention patterns to decrease hospital readmissions in older patients who have had a fall. Also, the included studies were recent, which underlines the fact that hospital readmissions are a relatively new concern for researchers and public health authorities.

\section{REFERENCES AND/OR ACKNOWLEDGEMENTS}

The authors thank Dr Clementz, Pr Kergoat, Mrs Bolduc, Dr Russell and Mrs Harper, who kindly agreed to provide us with additional data and allowed us to perform our systematic review.

No conflict of interest.

\section{PSQ-110 NUTRITIONAL ASSESSMENT IN A LONG TERM CARE FACILITY}

${ }^{1} \mathrm{~T}$ Rico-Gtierrez*, ${ }^{1} \mathrm{M}$ Vidal Iglesias, ${ }^{2} \mathrm{RA}$ Herrero Juarez, ${ }^{1} \mathrm{~J}$ Lázaro Cebas, ${ }^{1} \mathrm{C}$ Cáceres Velasco, ${ }^{1} \mathrm{MT}$ Criado Illana, ${ }^{2} \mathrm{~B}$ Cañuelo Coleto. ${ }^{1}$ Hospital General De Segovia, Pharmacy, Segovia, Spain; ${ }^{2}$ Residencia Mixta Personas Mayores, Doctor, Segovia, Spain

\subsection{6/ejhpharm-2020-eahpconf.427}

Background and importance A high risk of malnutrition is associated with decreased functionality and quality of life. Early identification of malnutrition risk by nutrition assessments plays an important role in the successful interventions in the elderly.

Aim and objectives To determine the nutritional status of residents in a long term care facility.

Material and methods A descriptive, observational, cross sectional study was completed during the month of March 2019. All patients in a long term care facility were included during that month, except for day and short term residents. Collected data were age, sex, weight, height, mid-arm and calf circumferences and body mass index (BMI).

MNA is a validated screening tool that is used to evaluate nutritional status in the elderly. It comprises two sections: screening with six questions with a maximum score of 14 (07, malnutrition; 8-11, risk of malnutrition; 12-14, normal nutritional status) and a full evaluation with 12 items up to a maximum score of 16 . The sum of both parts is the final score and establishes three ranges: $24-30=$ normal nutritional status, $17-23.5=$ at risk of malnutrition and $<17=$ malnutrition.

Both sections of the MNA were completed by all residents and information on the possible causes of malnutrition was provided. Serum albumin was also determined (normal value $3.4-4.8 \mathrm{~g} / \mathrm{dL})$.

Results

\section{Abstract 5PSQ-110 Table 1}

\begin{tabular}{lcc}
\hline $\mathbf{N}$ & $\mathbf{8 3}$ & Range 50-100 \\
\hline Mean age (years) & 86.9 & \\
Sex (\%) & & \\
Women & 75.9 & \\
Men & 24.1 & \\
Mean weight $(\mathrm{kg})$ & 58.7 & $95 \% \mathrm{Cl} 55.2-62.2$ \\
Mean height $(\mathrm{m})$ & 1.55 & $95 \% \mathrm{Cl} 1.53-1.57$ \\
Mean BMI $\left(\mathrm{kg} / \mathrm{m}^{2}\right)$ & 24.3 & $95 \% \mathrm{Cl} 23.1-25.4$ \\
\hline
\end{tabular}

\section{Abstract 5PSQ-110 Table 2}

\begin{tabular}{llll}
\hline Mean score & & & \\
\hline Screening & 11.48 & $95 \% \mathrm{Cl} 20.6-22.1$ & $\begin{array}{l}\text { Normal status } \\
8-11 \text { points }\end{array}$ \\
$\begin{array}{l}\text { Full evaluation } \\
\text { Final score }\end{array}$ & 10.50 & $95 \% \mathrm{Cl} 10.2-10.9$ & At risk of malnutrition \\
& 21.31 & $95 \% \mathrm{Cl} 20.6-22.1$ & $17-23.5$ points \\
$\begin{array}{l}\text { Mean serum } \\
\text { albumin (g/dL) }\end{array}$ & 3.50 & $95 \% \mathrm{Cl} 3.47-3.59$ & \\
\hline
\end{tabular}

